\title{
Continuous cropping of alfalfa (Medicago sativa L.) reduces bacterial diversity and simplifies cooccurrence networks in aeolian sandy soil
}

\author{
Yanxia Xu${ }^{1}$, Junjie Liu ${ }^{2,}{ }^{*}$, Xuefeng Liu ${ }^{1}$, Hong Li ${ }^{1}$, Zhao Yang ${ }^{1}$, Hongbao Wang ${ }^{1}$, Xinyu Huang ${ }^{1}$, Lan Lan ${ }^{1}$, \\ Yutong $\mathrm{An}^{1}$, Lujun $\mathrm{Li}^{2,3}$, Qin Yao', Guanghua Wang ${ }^{2}$
}

1 Branch of Animal Husbandry and Veterinary of Heilongjiang Academy of Agricultural Sciences, Qiqihar 230202, China

2 Key Laboratory of Mollisols Agroecology, Northeast Institute of Geography and Agroecology, Chinese Academy of Sciences, Harbin 130102, China

3 University of Chinese Academy of Sciences, Beijing 100049, China

H I G H L I G H T S

- 10 years of CC was a cut-off point in separating soil bacterial community structures.

- Soil $\mathrm{pH}$ and $\mathrm{P}$ were well associated with changes of diversity and community structures.

- $\mathrm{N}$ fixation bacteria were increased with successive year, but $P, K$ solubilizing bacteria decreased.

- Monocropped alfalfa simplified the complexity of the cooccurrence networks.

\section{ARTICLE INFO}

\section{Article history:}

Received October 12, 2020

Revised January 25, 2021

Accepted February 10, 2021

Keywords:

Aeolian sandy soil

Continuously cropped alfalfa

Cooccurrence networks

10 years

Functional bacteria
GRAPHICAL ABSTRACT

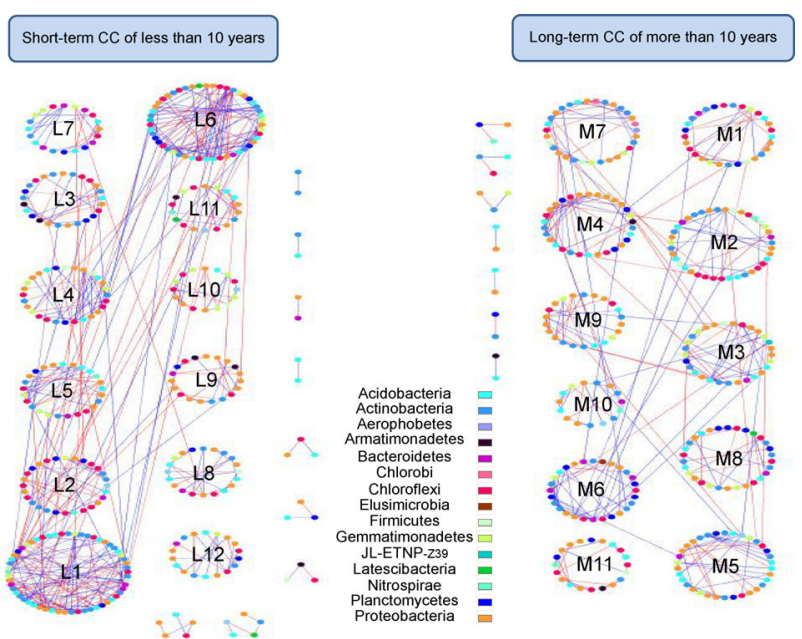

Alfalfa is a perennial herbaceous forage legume that is remarkably and negatively affected by monocropping. However, the contribution of the changes in bacterial communities to soil sickness in alfalfa have not been elucidated. Therefore, we investigated bacterial community structures in response to monocropped alfalfa along the chronosequence. Continuous cropping remarkably reduced bacterial alpha diversity and altered community structures, and soil $\mathrm{pH}$, total $\mathrm{P}$ and available $\mathrm{P}$ were strongly associated with the changes of bacterial diversity and community structures. Intriguingly, 10 years of monocropped alfalfa might be a demarcation point separating soil bacterial community structures into two obvious groups that containing soil samples collected in less and more than 10 years. The relative abundances of copiotrophic bacteria of Actinobacteria and Gammaproteobacteria significantly increased with the extension of continuous cropping years, while the oligotrophic bacteria of Armatimonadetes, Chloroflexi, Firmicutes and Gemmatimonadetes showed the opposite changing patterns. Among those altered phyla, Actinobacteria, Chloroflexi, 
Alphaproteobacteria and Acidobacteria were the most important bacteria which contributed $50.86 \%$ of the community variations. Additionally, the relative abundances of nitrogen fixation bacteria of Bradyrhizobium and Mesorhizobium obviously increased with continuous cropping years, while the abundances of Arthrobacter, Bacillus, Burkholderiaceae and Microbacterium with potential functions of solubilizing phosphorus and potassium remarkably decreased after long-term continuous cropping. Furthermore, bacterial cooccurrence patterns were significantly influenced by continuous cropping years, with long-term monocropped alfalfa simplifying the complexity of the cooccurrence networks. These findings enhanced our understandings and provided references for forecasting how soil bacterial communities responds to monocropped alfalfa.

(c) Higher Education Press 2021

\section{Introduction}

Continuous cropping of one kind of crop has frequently been shown to be an unsustainable agricultural practice, because it causes soil sickness and reduces crop yielding and quality (Liu et al., 2012; Xuan et al., 2012). Although the advantages of crop rotation and intercropping in improving soil nutrients and enhancing crop yields are well known, continuous cropping is still common in modern agricultural systems due to several factors, such as limited arable land, economic interests and climatic constraints (Liu et al., 2012). Numerous studies have revealed that continuous cropping leads to soil sickness involving the deterioration of soil physicochemical properties, a decline in soil enzyme activity, the enrichment of soil-borne plant pathogens, and the accumulation of autotoxic substances; these phenomena are considered to be soil sickness (Huang et al., 2013; Zhou et al., 2014). Studies focusing on these aspects have revealed that the abovementioned soil abiotic and biotic factors are closely associated with soil sickness, and these interrelated factors lead to the mechanisms underlying this decrease in yield being very complex (Li et al., 2010). Recently, studies focusing on revealing the mechanisms of soil sickness in grain crops, such as rice (Oryza sativa L.) (Xuan et al., 2012), soybean (Glycine max L.) (Liu et al., 2019), and corn (Zea mays L.) (Gentry et al., 2013) have been widely reported. However, research on the negative effects of continuous cropping systems on the feed crops is still rudimentary.

Alfalfa (Medicago sativa L.), a perennial herbaceous forage legume with high economic value, is widely cropped in the semiarid and arid regions of northern China (Zhang et al., 2016; Yao et al., 2019). It is often intercropped with different crops in the global scale to make a great contribution to maintaining soil organic carbon and accumulating nitrogen in soil (Bagavathiannan et al., 2011). In China, alfalfa has been cultivated annually for more than $4 \times 10^{6}$ hectares. To meet the forage, continuous cropping of alfalfa is a very common agricultural practice in northern China (Zhang et al., 2016). However, previous studies have revealed that alfalfa yield was reduced very seriously after eight to ten years of continuous cropping (Jiang et al., 2007; Li and Huang, 2008), and this decline was directly or indirectly associated with the changes in soil physical, chemical and biological properties (Ren et al., 2011). Thus, it becomes very important to reveal the mechanisms of how and to what extent continuous cropping of alfalfa affects its growth and health in terms of biotic factors, since previous research on this aspect has focused on the variation in soil physicochemical properties and its potential effect on alfalfa yield.

Soil microorganisms play a crucial role in biogeochemical cycling, which is closely related to soil fertility as well as plant health (Prosser, 2019). Multiple studies have revealed that several factors, including soil physicochemical properties, the abundance of plant species and diversity and different cropping systems (continuous cropping, intercropping and no-tillage etc.), significantly contribute to regulating microbial community structures (Rosenzweig et al., 2012; Zhou et al., 2014). For instance, previous studies have shown that the abundance and diversity of both the bacterial and fungal communities were significantly changed in response to continuous cropping, which led to a decrease in bacterial abundance but increased fungal abundances in both bulk and rhizospheric soils (Liu et al., 2017; 2019). The results were consistent with a large body of literature supporting the view that continuous cropping breaks the balance of original soil microorganisms, and this phenomenon can be described as the change from "a high nutrient bacterial type soil" to "a low nutrient fungal type soil" (Xuan et al., 2012; Xiong et al., 2015). Among the shifts in fungal communities, the relative abundance of soil-borne plant pathogens, especially Fusarium sp. was significantly increased in continuous cropping systems, which has been widely reported in many previous studies ( $\mathrm{Li}$ and Liu, 2019). However, the variation of bacterial taxa in response to continuous cropping varied among studies due to the variety of research methods, soil types and the monocropping years based on a meta-analysis (Venter et al., 2016). In addition, whether the shifts of soil bacterial community structures responds to continuous cropping is consistent between annual plants and perennial crops has not been documented.

A comprehensive study to evaluate the effect of soil microbial communities in influencing crop productivity had documented that the impacts on plant height, biomass and yield in response to continuous cropping were mainly due to biological characteristics rather than nonbiological factors (Wei et al., 2015). Therefore, a study focusing on the changes in biological factors in response to continuous cropping, can provide insight into the mechanisms of how bacterial communities are influenced and the potential connections between these impacts and continuous cropping systems. In our previous study, we investigated the effects of different years of continuously cropped alfalfa on the fungal communities in north east China using a high-throughput sequencing method, 
and we observed that the increase in the relative abundances of several soil-borne plant pathogens, such as Cyphellophora sp. and Haematonectria hematococca were associated with continuous cropping sickness after monocropped alfalfa (Yao et al., 2019). Nevertheless, studies related to shifts in bacterial communities in alfalfa fields are limited (Zhong et al., 2012; Luo et al., 2018). In this study, the diversity, structure and ecological network characteristics of bacterial communities in continuously cropped alfalfa along the chronosequence were examined. The aims of this research were (1) to evaluate the dynamic changes in soil bacterial diversity, components and structures with different continuous cropping years; (2) to reveal the relative contributions of soil factors in shaping bacterial communities to estimate the biotic barrier mechanisms for continuous cropping of alfalfa; and (3) to examine bacterial network structures and putative keystone species in response to short-term and long-term continuous cropping years.

\section{Materials and methods}

\subsection{Study site and soil sampling}

The field experiment was established in 1979 to assess the impacts of continuously cropped alfalfa in the Heilongjiang Institute of Animal Husbandry in Qiqihar city $\left(47^{\circ} 15^{\prime} \mathrm{N}\right.$, $123^{\circ} 41^{\prime} \mathrm{E}$ ), China. The mean annual temperature is $3^{\circ} \mathrm{C}$, and the mean annual precipitation is $450 \mathrm{~mm}$. The soils used in this study are aeolian sandy soils in the semiarid regions with a mean soil $\mathrm{pH}$ is above 7.0 , and the salinity of approximately $0.24 \%$.

The widely planted varieties of Medicago sativa L. CV. Longmu801 were seeded for each treatment that covered more than $900 \mathrm{~m}^{2}$ in area. The compound chemical fertilizer containing $\mathrm{N} 16 \%, \mathrm{P}_{2} \mathrm{O}_{5} 16 \%$ and $\mathrm{K}_{2} \mathrm{O} 16 \%$, which was annually applied at $280 \mathrm{~kg} \mathrm{ha}^{-1}$ in each plot in May. The management of the alfalfa fields was conducted to the conventional tillage practice without grazing. Alfalfa was mowed from soil surface twice a year in June and August and removed from the field. The soils were collected from alfalfa fields with continuously cropped alfalfa for 1, 2, 6, 9, 12, 13 and 35 years (encoded as ACC1y, 2y, 6y, 9y, 12y, 13y and $35 y$ ) on June 25,2015 . Specifically, no less than 8 individual soil cores (0-15 cm soil depth) were randomly collected and merged into a repeated soil sample to minimize the within-plot variations. Overall, 21 soil samples were obtained from seven continuous cropping alfalfa fields with three replicates in each treatment. Each soil sample was put into a $50 \mathrm{~mL}$ centrifuge tube and kept in a $-80^{\circ} \mathrm{C}$ freezer for the extraction of soil total DNA. The other soil samples were air-dried for measuring soil chemical properties, and the results are shown in Table S1.

\subsection{Soil DNA extraction and Illumina MiSeq sequencing}

The soil DNA used in this study was in line with our previous studies, and was extracted with the Fast DNA ${ }^{\circledR}$ SPIN Kit for Soil (MP Biomedicals, USA) (Yao et al., 2019).

The bacterial 16S rRNA gene of the V4-V5 hypervariable regions was amplified using F515/R907 (Biddle et al., 2008). A modification of adding a unique 8-nt barcode sequence to the forward and reversed primers to distinguish the amplified products. PCRs were conducted using a $25 \mu \mathrm{L}$ mixture containing $1.0 \mu \mathrm{L}$ of template DNA, $0.5 \mu \mathrm{L}(10 \mu \mathrm{M})$ of each primer, and $23 \mu \mathrm{L}$ of Platinum PCR SuperMix (TransGen Biotech Co. Ltd., Beijing, China). PCR amplification cycling was initial denaturation at $95^{\circ} \mathrm{C}$ for $5 \mathrm{~min}$, followed by 30 cycles $\left(95^{\circ} \mathrm{C}\right.$ for $1 \mathrm{~min}, 63^{\circ} \mathrm{C}$ for $1 \mathrm{~min}, 72^{\circ} \mathrm{C}$ for $1 \mathrm{~min}$ ) with a final extension at $72^{\circ} \mathrm{C}$ for $5 \mathrm{~min}$. Each PCR product was purified and pooled in equimolar amounts, and then prepared for sequencing using an Illumina MiSeq platform (Majorbio BioPharm Technology Co., Ltd. Shanghai, China).

\subsection{Sequence data analysis}

The raw FASTQ data were demultiplexed and quality-filtered using QIIME Pipeline Version 1.9.0. FLASH software was used to join the paired reads (Magoč and Salzberg, 2011). Then, the low-quality sequences were removed from further analysis if they did not meet the following criteria: sequences were shorter than $200 \mathrm{bp}$, and/or the score of average base quality was less than 30 . The trimmed sequences were chimera-detected and removed with the Uchime algorithm (Edgar et al., 2011). The sequences of high quality were clustered into operational taxonomic units (OTUs) with $97 \%$ similarity using the UPARSE pipeline. A representative sequence of each phylotype was selected and aligned using a Python Nearest Alignment Space Termination (PyNAST) tool, and then a phylogenetic tree was built using Fast Tree after the alignment of each phylotype (Price et al., 2009). The taxonomic information of each OTU was obtained by RDP Classifier at a confidence threshold of 0.80 . The OTUs that was not assigned as bacteria were filtered out for further analysis. The raw $16 \mathrm{~S}$ rRNA sequences have been submitted to the NCBI Sequence Read Archive with accession number of SRP234494.

\subsection{Network construction and analysis}

The phylogenetic Molecular Ecological Networks (pMENs) method was employed to construct bacterial networks based on the Random Matrix Theory (RMT) methods (http://ieg2.ou. edu/MENA/main.cgi). In brief, a prepared OTU file with the relative abundance of each OTU was higher than $0.01 \%$, and only those occurring in more than half of all soil samples were uploaded to create each network. Two individual bacterial networks within the samples from less (L10) or more (M10) than 10 years of continuous cropping were obtained with automatically generated cutoff values (similarity threshold, $S_{t}$ ). A node represents the OTU, and a link between the pair of each OTU was determined if the correlation between their abundance exceeded the $S_{t}$ with a $p$ value lower than 0.01 in a 
network. For information on the algorithms, the network theories and operational procedures, refer to Deng et al. (2012). A standard $t$ test was then performed to assess the significance of network indices between L10 and M10 of continuous cropping of alfalfa (Deng et al. 2012). The network graphs were visualized with Cytoscape 2.8.2 software.

\subsection{Statistical analysis}

To compare the relative variation among samples, a subset of 20000 sequences in each soil sample was randomly selected and performed for alpha- and beta-diversity analyses. The indices of alpha-diversity such as Chao1, Shannon, Simpson, and phylogenetic diversity (Faith's PD) were performed in QIIME and were used to compare the differences between samples. One-way analysis of variance (ANOVA) was used to analyze the significant differences in alpha-diversity and the relative abundances of different taxonomic levels at the phylum, genus and OTUs levels of bacterial communities. In addition, SPSS software was used to analyze Spearman's correlation coefficient to reveal the possible correlations between the number of continuous cropping years, the relative abundances of bacterial communities at different taxonomic levels, alpha-diversity and soil chemical properties. Principal coordinate analysis (PCoA) and clustering analysis of soil bacterial community structures based on UniFrac metrics and Bray-Curtis distance, respectively, were used to identify the differences in bacterial community structures among soil samples. Mantel tests was performed to assess the links between soil factors and bacterial communities. The soil factors associated with bacterial communities were used to construct the soil property matrix for canonical correspondence analysis (CCA) and variation partitioning analysis (VPA). To identify which bacterial phyla make a great contribute to bacterial community dissimilarity between samples, SIMPER analysis was used to uncover the relative contribution of bacteria at each phylum/class level to the total community variation between-treatment variations. Mantel tests, CCA, VPA and SIMFER analysis were conducted using the "vegan" package in the $\mathrm{R}$ environment ( $\mathrm{R}$ Development Core Team, 2010).

\section{Results}

\subsection{Soil chemical properties}

Most of the soil chemical properties were remarkably changed with different years of continuous cropping with the exception of $\mathrm{NH}_{4}{ }^{+}-\mathrm{N}$ (Table S1). A simple linear regression analysis showed that soil $\mathrm{H}_{2} \mathrm{O} \%(r=0.445, P=0.043)$, and $\mathrm{AK}$ ( $r=0.665, P=0.001)$ were significantly and positively related to the continuous cropping year, while C:N $(r=-0.552$, $P=0.009)$, TP $(r=-0.740, P<0.0001)$ and AP $(r=-0.518$, $P=0.016$ ) were negatively correlated. In addition, soil $\mathrm{pH}, \mathrm{TC}$, $\mathrm{TN}, \mathrm{TK}$ and AN fluctuated with the continuous cropping year, with soil $\mathrm{pH}(8.60)$ and TK $\left(21.46 \mathrm{~g} \mathrm{~kg}^{-1}\right)$ reaching a peak in ACC9y, whereas TC $\left(24.01 \mathrm{~g} \mathrm{~kg}^{-1}\right)$, TN $\left(1.44 \mathrm{~g} \mathrm{~kg}^{-1}\right)$, and AN (109.23 $\mathrm{mg} \mathrm{kg}^{-1}$ ) contents were the lowest in ACC9y.

\subsection{Bacterial diversity and structures}

In total, 667287 high quality sequences were obtained from 21 soil samples, and 20056-36369 sequences per soil sample (mean $=27813$ ). Of these obtained sequences, 4227 different OTUs were detected across all samples, ranging from 1994 in ACC12y to 2134 in ACC1y. The coverage index in each sample was estimated to be up to $95 \%$. The Shannon and Chao 1 indices were remarkably higher in the initial cropping years of ACC1y and ACC2y (Table S2). Regression analysis revealed that OTU richness $(r=-0.502$, $P=0.020)$ and phylogenetic diversity $(r=-0.487, P=0.025)$ were significantly and negatively correlated with cropping year. In addition, pairwise analysis showed that OTU richness $(r=-0.556, P=0.009)$ and phylogenetic diversity $(r=-0.492$, $P=0.023$ ) were significantly and negatively correlated with soil $\mathrm{pH}$; furthermore, phylogenetic diversity $(r=0.519$, $P=0.016)$ was positively correlated with soil TP content (Fig. 1). Other soil parameters had no significant correlation with bacterial richness and diversity (Table S3).

Principal coordinate analysis (PCoA) showed that all soil samples were well divided from each other, indicating that bacterial community structures were significantly different among treatments (Fig. 2A). Although the ACC12y and ACC13y treatments had no relatively distinguishing boundaries, the perMANOVA tests $(p=0.028)$ showed that the community structure was significantly different between these two treatments. Overall, all soil samples in the plot were clearly separated into two major groups, which containing the soil samples collected in less and more than 10 years of continuous cropping, respectively (perMANOVA tests, $p<0.001$ ) (Fig. 2A). This grouping pattern was further confirmed by hierarchical clustering based on the Bray-Curtis matrix (Fig. 2B).

SIMPER analysis was used to evaluate the relative contribution of each bacterial phylum/class level to the total community variation. Based on the bacterial communities being clustered into two major groups, here, the relative abundance of bacteria in the less or more than 10 years were calculated together. The results revealed that Actinobacteria, Chloroflexi, Acidobacteria and Alphaproteobacteria contributed $50.86 \%$ of the bacterial community variations, among these changed phyla, Actinobacteria contributed $21.34 \%$ which was the primary member in shaping the variation. In addition, although the relative abundance of Alphaproteobacteria was higher than Chloroflexi, it made a relatively smaller contribution to the community variation than Chloroflexi (Fig. 3).

\subsection{Bacterial community compositions}

Thirty-six bacterial phyla were observed across all the 

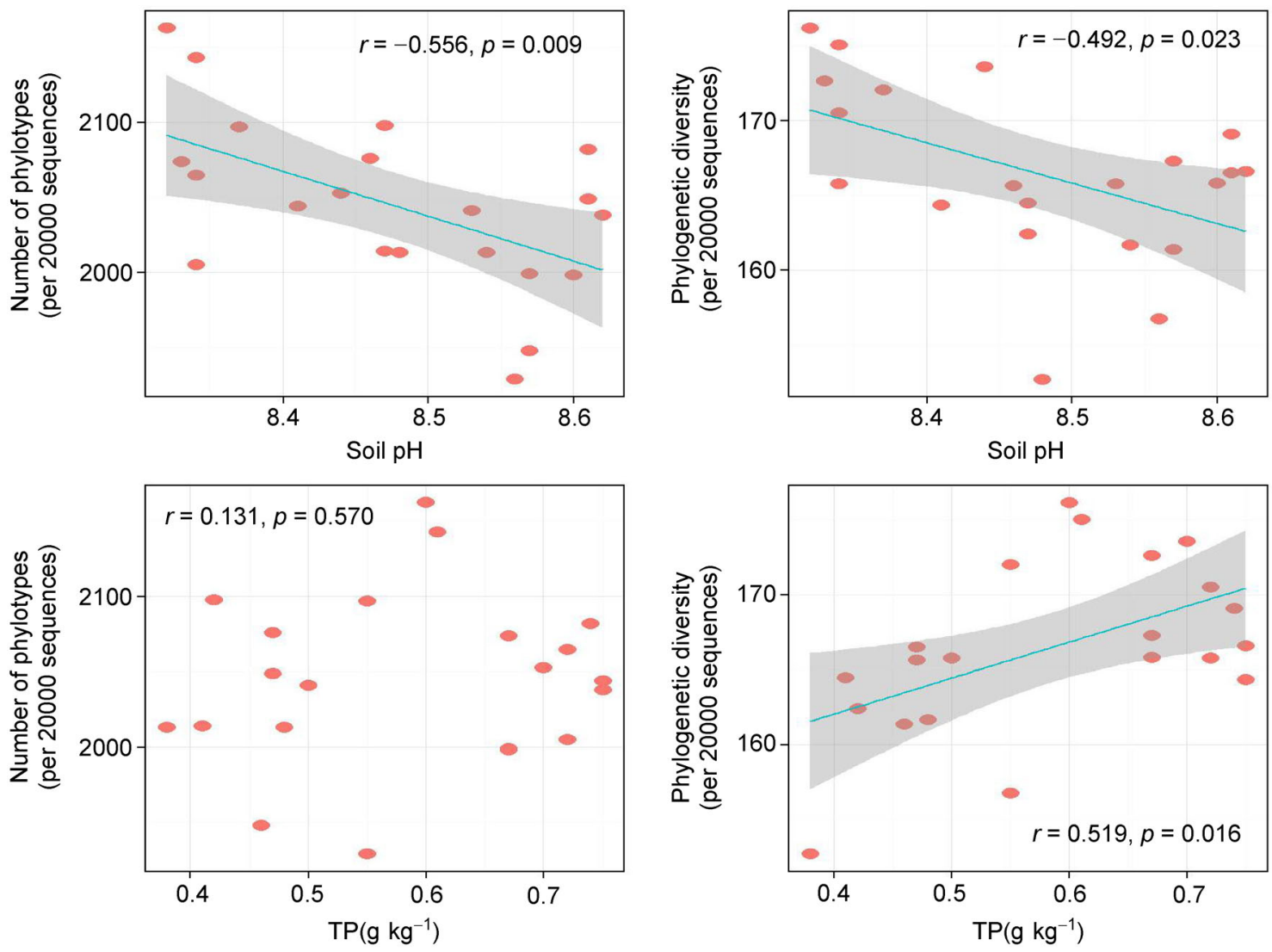

Fig. 1 Spearman's correlation coefficient between soil bacterial phylotype richness, phylogenetic diversity and soil pH and TP.
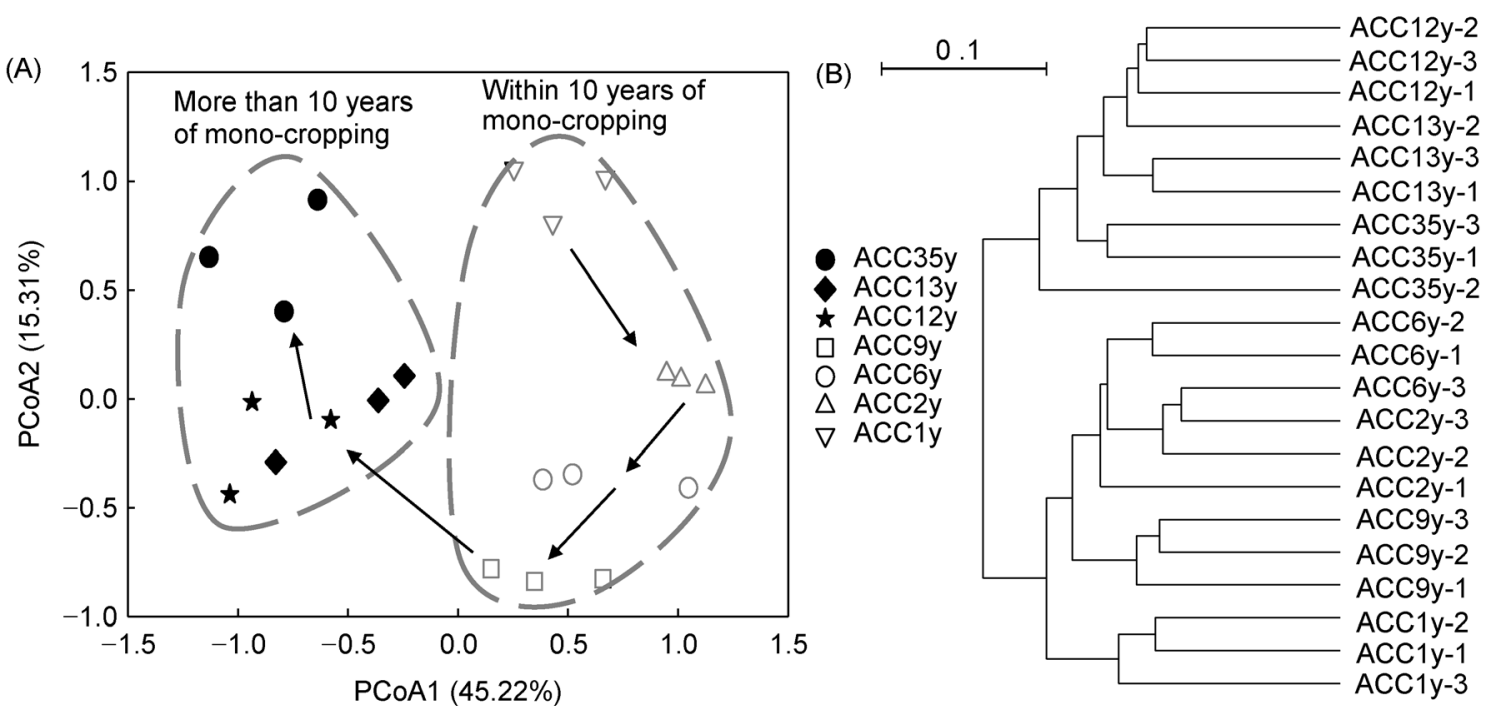

Fig. 2 Principal coordination analysis $(A)$ and clustering analysis $(B)$ of soil bacterial community structures based on UniFrac metrics and Bray-Curtis distance. The trajectory succession of bacterial communities along with cropping years is shown with arrows in Fig. 2A. 


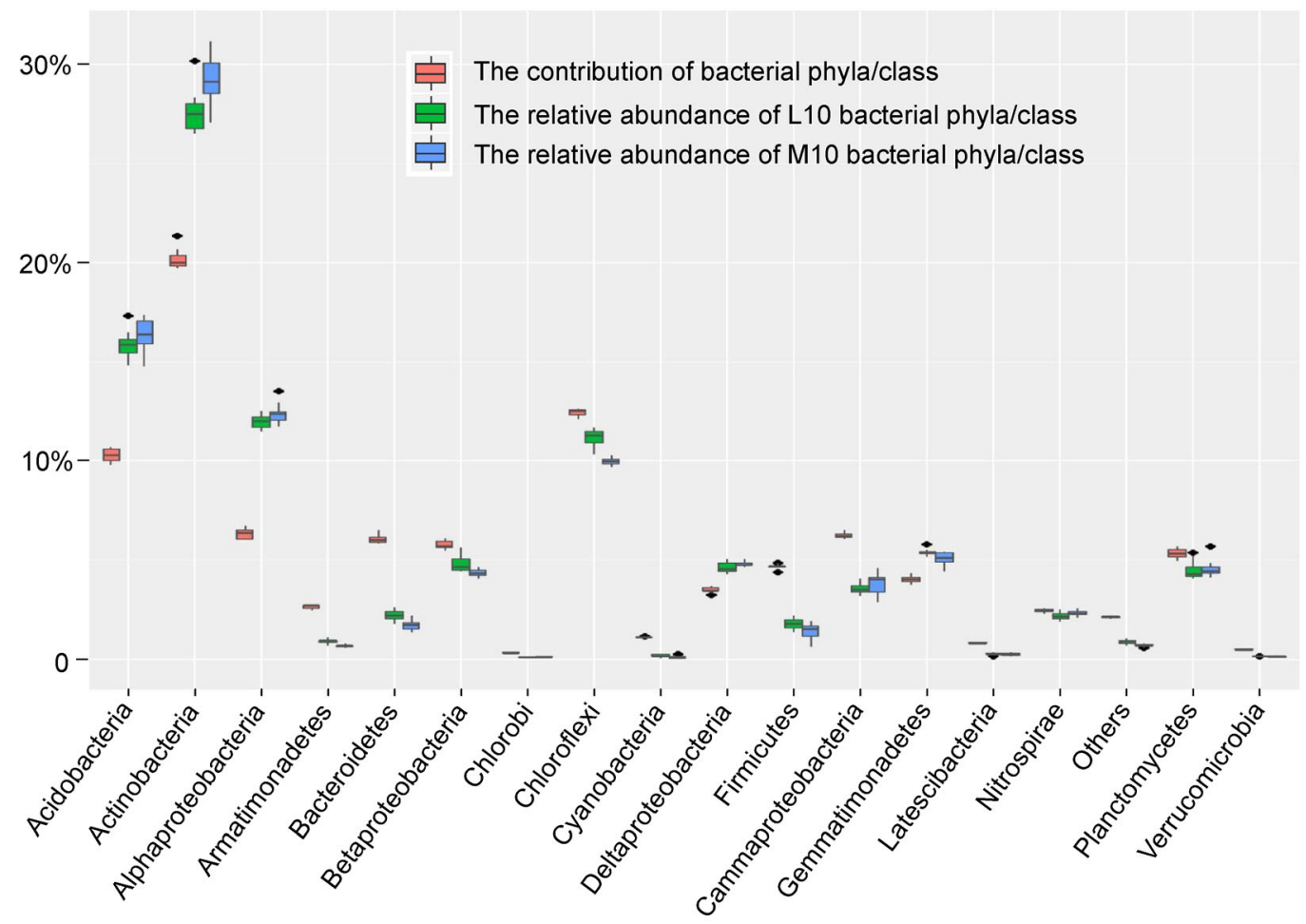

Fig. 3 The relative abundance and contribution of the bacterial phyla and/or classes, which generated the community variations between less (L10) and more than 10 years (M10) of continuously cropped alfalfa, respectively.

samples, among which the dominant bacterial phyla with relative abundances higher than $5 \%$ were Actinobacteria (28.16\%), Proteobacteria (25.17\%), Acidobacteria (16.23\%), Chloroflexi (10.52\%) and Gemmatimonadetes (5.34\%), which accounted for $85.42 \%$ of the total obtained sequences. The phyla of Armatimonadetes, Bacteroidetes, Chlorobi, Cyanobacteria, Firmicutes, Latescibacteria, Nitrospirae, Planctomycetes, Thaumarchaeota and Verrucomicrobia were detected in all of the soil samples, with the relative abundances were higher than $1 \%$. In addition, 21 rare phyla were sporadically identified in most of the soil samples (Table S4).

One-way ANOVA revealed that the relative abundance of some bacterial phyla was significantly shifted among soil samples. Overall, the relative abundances of Actinobacteria and Gammaproteobacteria were significantly positively correlated with cropping year, while Armatimonadetes, Chloroflexi, Firmicutes and Gemmatimonadetes were negatively correlated (Fig S1). Additionally, several soil properties were also significantly positively and/or negatively correlated with the relative abundance of the main bacterial groups (Table S5).

More than 540 bacterial genera were detected, and 149 and 74 genera were obtained with average relative abundance greater than $0.1 \%$ and/or $0.3 \%$, respectively. Among them, Acidobacteria_subgroup 6, Gaiellales, Gemmatimonadaceae, JG30-KF-CM45, Actinobacteria_norank, Nitrospira and Arthrobacter were the most abundant genera across all of the soil samples, with average relative abundances of $9.74 \pm 0.58 \%, 4.21 \pm 0.82 \%, 3.89 \pm 0.37 \%, 2.72 \pm 0.41 \%$, $2.45 \pm 0.40 \%, 2.28 \pm 0.20 \%$ and $1.98 \pm 0.72 \%$, respectively
(Table S4). The relative abundance of Bradyrhizobium, Gaiella, Mesorhizobium, Xanthobacteraceae, etc., 20 bacterial genera was significantly increased with continuous cropping years, while 11 bacterial genera, including Arthrobacter, Bacillus, Blastococcus, Microbacterium, Nocardioides, Rhodospirillales, etc., decreased with the extension of cropping years $(P<0.05)$. In addition, those genera mentioned above were also significantly positively or negatively correlated with the soil properties (Table S5).

\subsection{Bacterial community structures related to soil factors}

The Mantel test results revealed that the community structures were significantly affected by soil $\mathrm{pH}, \mathrm{TP}, \mathrm{AP}, \mathrm{NO}_{3}{ }^{-} \mathrm{N}$ and $\mathrm{C}: \mathrm{N}$ (Table 1). These correlative soil properties were selected and analyzed by CCA and VPA. Similar to the PCoA plot, the CCA analysis showed that bacterial community was significantly affected by successional cropping year. Soil $\mathrm{pH}$ was positively related to soil samples collected within 10 years, while TP and AP were positively related to soil samples collected after more than 10 years of continuous cropping (Fig. 4A). In addition, a VPA analysis showed that these soil properties together explained $54.83 \%$ of the bacterial community variations, leaving $45.17 \%$ of the unexplained variation. Among these related soil parameters, soil $\mathrm{pH}$, TP, AP, $\mathrm{NO}_{3}{ }^{-} \mathrm{N}$ and $\mathrm{C}: \mathrm{N}$ explained $13.54 \%, 8.46 \%, 4.61 \%, 4.41 \%$ and $3.78 \%$ of the bacterial community variations, respectively (Fig. 4B). 
(A)

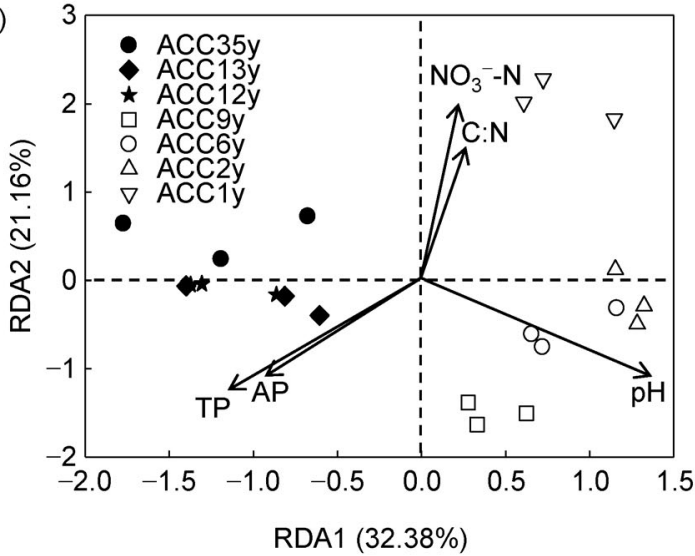

(B)

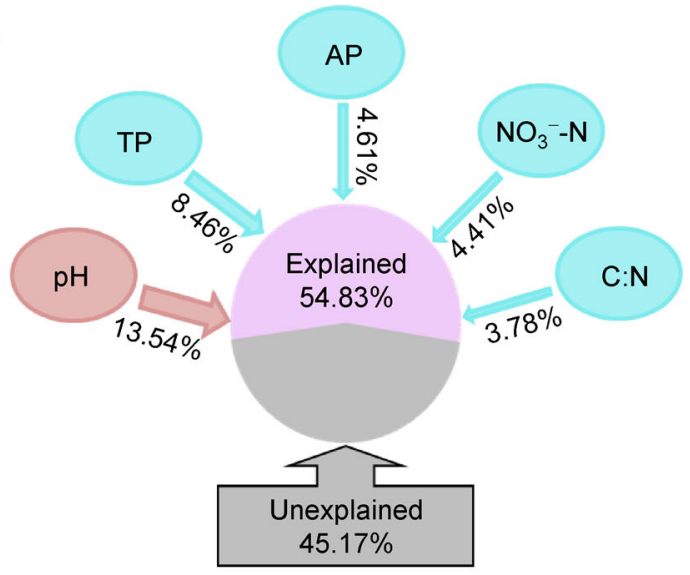

Fig. 4 Canonical correspondence analysis (CCA) (A), and the variation partition analysis (VPA) (B) of soil variables to explain the variations in bacterial community structures.

Table 1 Mantel test results show the correlation between bacterial community structures and soil variables.

\begin{tabular}{lll}
\hline Variable $^{a}$ & $R$ & $P$ \\
\hline $\mathrm{pH}$ & $\mathbf{0 . 4 4 1}$ & 0.001 \\
$\mathrm{TP}$ & $\mathbf{0 . 3 6 4}$ & 0.002 \\
$\mathrm{AP}$ & $\mathbf{0 . 2 5 3}$ & 0.005 \\
$\mathrm{NO}_{3}{ }^{-} \mathrm{N}$ & $\mathbf{0 . 2 1 6}$ & 0.028 \\
$\mathrm{C}: \mathrm{N}$ & $\mathbf{0 . 1 8 9}$ & 0.038 \\
$\mathrm{H}_{2} \mathrm{O} \%$ & 0.173 & 0.051 \\
$\mathrm{AK}$ & 0.169 & 0.063 \\
$\mathrm{TN}^{-}$ & 0.163 & 0.064 \\
$\mathrm{AN}$ & 0.133 & 0.093 \\
$\mathrm{NH}_{4}{ }^{+}-\mathrm{N}$ & -0.106 & 0.167 \\
$\mathrm{TK}^{\mathrm{TC}}$ & -0.059 & 0.532 \\
\hline
\end{tabular}

a TC, TN, TP and TK indicate soil total carbon, nitrogen, phosphorus and potassium, respectively; AN, AP and AK indicate soil available nitrogen, phosphorus and potassium, respectively; $\mathrm{NH}_{4}{ }^{+}-\mathrm{N}$, ammonium nitrogen, $\mathrm{NO}_{3}{ }^{-} \mathrm{N}$, nitrate nitrogen. Values in bold indicate significant correlation $(P<0.05)$.

\subsection{Network analysis of bacterial communities}

The network structure of pMENs was constructed to reveal the interactions of bacterial community cooccurrence within the samples from less (L10) or more (M10) than 10 years of continuous cropping (Fig. 5). In general, the values of connectivity were well-fitted to the power-law patterns, with $R^{2}$ value of 0.81 and 0.91 in $L 10$ and $M 10$, respectively. The average geodesic distance (GD) and the average clustering coefficient (avgCC) in empirical networks were significantly higher than those in the corresponding random networks, suggesting that the overall networks fit for the typical smallworld and/or the modular characteristics. The values of the total nodes, links and the average connectivity (avgK) were remarkably increased in L10, and the average geodesic distance was significantly lower in M10 than in L10 (Table 2).

The topological roles of the individual node identified between two networks are shown as within module connectivity $(Z i)$ and among-module connectivity $(P i)$ plots (Fig. S2). The majority of the nodes with $97.6 \%$ of the L10 and M10 treatments were peripherals, making their links mostly inside their corresponding modules. Two (OTU1404 and OTU4163) of the six module hubs under L10 belonged to Chloroflexi which are closely related to Ktedonobacteria and uncultured bacteria, respectively, while the others belonged to different major taxa including Microlunatus, Myxococcales bacterium, Ohtaekwangia and Tumebacillus. In contrast, two (OTU 293 and OTU2265) of the five module hubs under M10 belonged to Acidobacteria, which had higher identity with subgroup 6 and Actinomycetales, while two module hubs (OTU3570 and OTU1580) were assigned as Proteobacteria phyla and belonged to Acetobacteraceae and Nannocystis. In addition, five OTUs belonging to Micromonosporaceae, Planctomyces, Roseiflexus, Gemmatimonadaceae and Planctomycetales playing as connectors were found in the L10 treatment. Interestingly, four of the eight connector OTUs (OTU756, OTU2289, OTU293 and OTU135) were derived from Actinobacteria, which are close to Nocardioides, Blastococcus, Actinomycetales and Amycolatopsis in the M10 treatment. Meanwhile, two connector OTUs (OTU3774 and OTU1168) belonging to Chloroflexi that are closely related to P2-11E and Anaerolineales, with the Gemmatimonadaceae and Planctomycetaceae genera, were categorized as connectors under M10 treatment. Furthermore, no overlapping genera of the module hub genes or connectors were found between the two treatments (Table S6). 


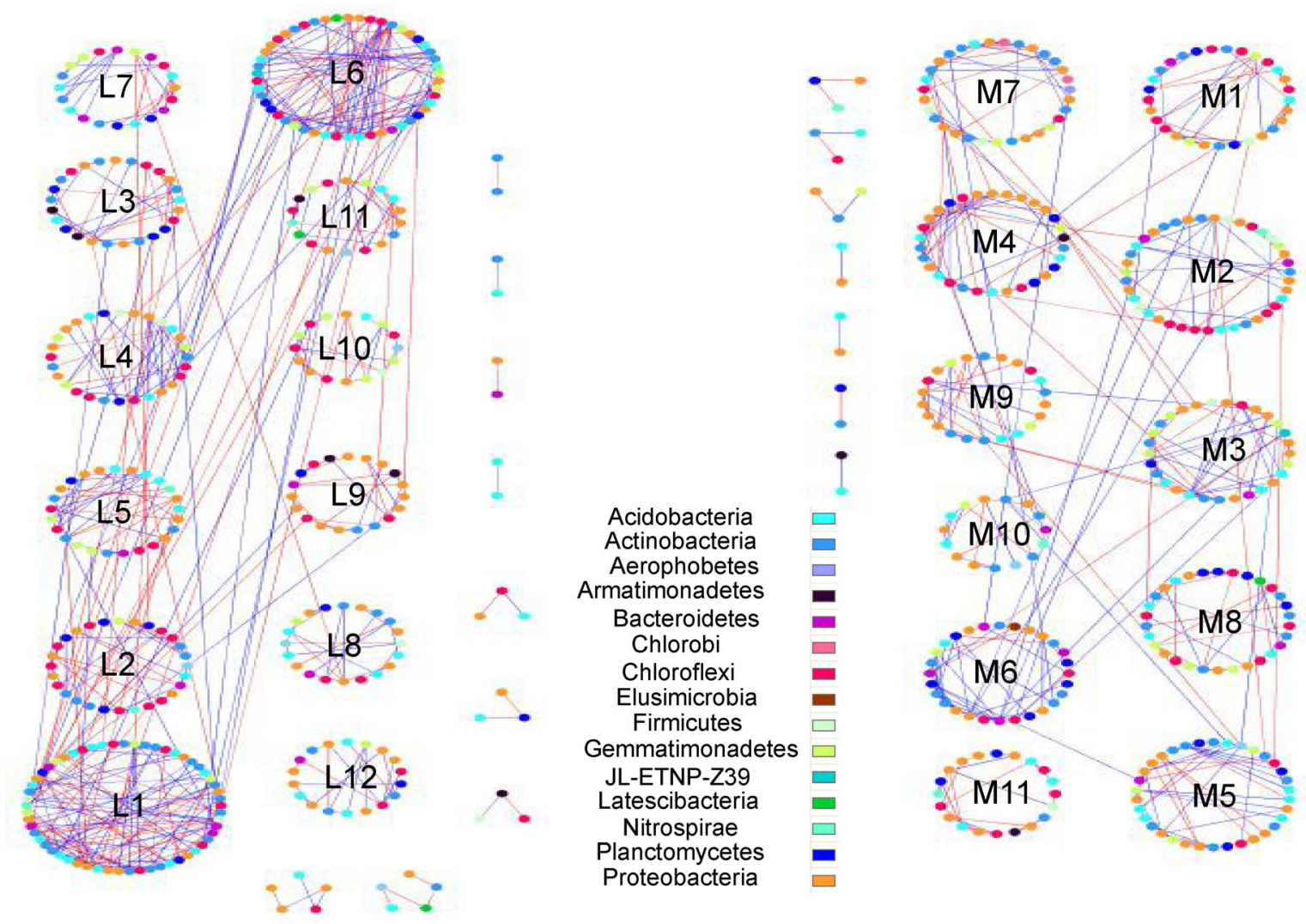

Fig. 5 Bacterial ecological networks displayed at the OTU level in less (L10) and more than 10 years (M10) of continuously cropped alfalfa, respectively. The blue and red lines represent negative and positive interactions between two relevant nodes. The letters of $L$ and $M$ indicate the soil samples that were collected in less and more than 10 years of continuous cropping of alfalfa. The numbers after the letters of $L$ and $M$ represent the different network modules.

Table 2 Topological properties of the Molecular Ecological Networks (MENs) of bacterial communities in less (L10) and more than 10 years (M10) of continuously cropped alfalfa, and their associated with random pMENs.

\begin{tabular}{|c|c|c|}
\hline Treatments & M10 & L10 \\
\hline \multicolumn{3}{|l|}{ Empirical networks } \\
\hline No. of original OTUs & 670 & 670 \\
\hline Similarity threshold $\left(S_{t}\right)$ & 0.91 & 0.91 \\
\hline Network size ${ }^{a}(n)$ & 633 & 670 \\
\hline Links & 741 & 787 \\
\hline Avg. connectivity(avgK) & $2.24^{c}$ & $2.50^{\mathrm{c}}$ \\
\hline Avg. geodesic distance (GD) & $9.14^{\mathrm{c}}$ & $8.85^{c}$ \\
\hline Avg. clustering coefficient (avgCC) & $0.14^{\mathrm{c}}$ & $0.17^{\mathrm{c}}$ \\
\hline Modularity (M) & 0.87 & 0.88 \\
\hline$R^{2}$ of power-law & 0.81 & 0.91 \\
\hline \multicolumn{3}{|l|}{ Random networks ${ }^{b}$} \\
\hline Avg. geodesic distance (GD) $\pm S D$ & $7.210 \pm 0.167$ & $6.923 \pm 0.147$ \\
\hline Avg clustering coefficient (avgCC) $\pm S D$ & $0.003 \pm 0.002$ & $0.004 \pm 0.003$ \\
\hline Modularity $(M) \pm S D$ & $0.569 \pm 0.007$ & $0.569 \pm 0.005$ \\
\hline
\end{tabular}

\footnotetext{
${ }^{a}$ Number of OTUs (e.g., nodes) in a network. ${ }^{b}$ Random networks generated by rewiring of the links of a MEN into identical numbers of nodes and links to their corresponding empirical MEN. ${ }^{\mathrm{C}}$ Significant difference $(P<0.001)$ between M10 and L10 bacterial networks.
} 


\section{Discussion}

\subsection{Long-term continuous cropping of alfalfa reduced bacterial diversity}

Mounting evidence has revealed that continuous cropping has an adverse effect on microbial community diversity (Liu et al., 2014; Tsiafouli et al., 2015). However, the decreased soil microbial diversity was found between continuous cropping and rotation systems, and the changing patterns of microbial diversity along with different continuous cropping years had no consistent rules. For example, it was found that soil bacterial diversity significantly decreased (Liu et al., 2014), had no change (Zhou et al., 2014), and fluctuated as soil bacterial diversity increased after 20 years (Zhu et al., 2014) for monocropped potato (Solanum tuberosum L.), cucumber (Cucumis sativus L.) and soybean (Glycine max L.) under successional continuous cropping years, respectively. It should be noted that the crops mentioned above are annual crops. In this study, we found that both OTU richness and phylogenetic diversity were remarkably and negatively correlated with alfalfa continuous cropping years, which was in line with other perennial crops such as Jerusalem artichoke (Helianthus tuberosus L.) (Zhou et al., 2018), coffee (Zhao et al., 2018) and Panax notoginseng (Tan et al., 2017). Therefore, the bacterial community in monocropped perennial crops exposed to root exudates of the same crop is more susceptible to successive cropping obstacles than annual plants, which leads to a continuously decrease in bacterial diversity with continuous cropping years (Xiong et al., 2015; Chen et al., 2018).

The results of soil bacterial phylogenetic diversity and phylotype richness were negatively correlated with soil $\mathrm{pH}$, which is consistent with Chu et al. (2010), and Fierer and Jackson (2006), who reported that the correlation between bacterial alpha-diversity and soil $\mathrm{pH}$ is unimodal, while it was highest at neutral $\mathrm{pH}$ at the continental scale. Thus, the increas in soil $\mathrm{pH}$ caused by continuous cropping in alkaline soil may have negative roles in regulating soil bacterial diversity. Intriguingly, our previous study revealed that soil fungal phylogenetic diversity and phylotype richness were remarkably increased with the extension of continuous cropping years (Yao et al., 2019), which suggested that the response of soil bacterial and fungal diversity to continuous cropping years are fundamentally different (Bai et al., 2015 Liu et al., 2019). In addition, we observed that bacterial phylogenetic diversity was significantly positively correlated with soil TP, and thus, the decrease in soil TP content after long-term continuous cropping of alfalfa could inhibit microbial diversity and activity (Cleveland and Liptzin, 2007).

4.2 Changes in bacterial community structures induced by long-term continuous cropping

In this study, we observed that there were significant variations in soil bacterial community structures between each soil sample with different cropping years (perMANOVA tests, $p<0.05$ ). Intriguingly, both the PCoA and clustering analysis showed that bacterial communities were remarkably separated into two major groups, including soil samples collected in less and more than 10 years of continuously cropped alfalfa (Fig. 2). These changing patterns of bacterial community structures were in line with fungal communities under the same soil samples in our previous study (Yao et al., 2019), which reconfirmed that 10 years might be a demarcation point in the separation of both bacterial and fungal community structures under continuous cropping of alfalfa. Similarly, Zhang et al. (2013) found that the bacterial community subjected to 10 years of continuously cropped cotton formed new stable community structures under longterm stress from the external environment. Although alfalfa biomass and yield have not been determined in the current study, other studies have demonstrated that the intensive cultivation of alfalfa caused severe soil degradation and significantly limited production after 8 to 10 years in dryland and semiarid regions of north-western China and the Loess Plateau of China, respectively (Jiang et al., 2007; Li and Huang, 2008). Thus, the combination of the evidence with yield changes in previous reports, and the extent of correlation between the changes in soil properties, bacterial diversity and structures and the length of the alfalfa growth phase in this study, illustrate the roles of plant-microbe interactions in regulating the compositions of the bacterial communities by root turnover and root secretion of perennials (Strickland et al., 2009).

Soil pH and TP, AP contents were the key soil factors in shifting bacterial community structures in continuously cropped alfalfa fields (Table 1), whereas the soil $\mathrm{pH}$, TP and AP were positively related to soil samples collected in less and more than 10 years of continuously cropped systems, respectively (Fig. 4A). In general, the narrow soil pH range even at a small variance is still the principal factor in determining bacterial communities, which have been widely reported at the regional-scale culture systems (Degrune et al., 2015; Liu et al., 2017). Since bacteria play an essential role in soil $P$ dynamics, $P$ was reported to exert the obvious effects on bacterial community structures during continuous cropping (Udom and Ogunwole, 2015; Liu et al., 2019). In addition, soil TP and AP were reported as limiting factors in the production of perennial alfalfa in long-term continuous cropping systems (Jiang et al., 2007; Li and Huang, 2008). Thus, we speculated that soil bacterial communities may play a central role in soil $P$ dynamics to increase $P$ availability, especially after 10 years of successive cropping of alfalfa.

4.3 Variation in soil bacterial community compositions under long-term continuous cropping of alfalfa

Soil sickness induced by consecutive cropping can be partially caused by the changes in microbial community diversity and/or structures (Huang et al., 2018). However, the 
diversity may play less consequential roles than the specific and functional bacterial groups (Banerjee et al., 2016). In this study, the relative abundances of some copiotrophic bacteria of Actinobacteria and Gammaproteobacteria significantly increased with continuous cropping years and were positively correlated with TN content, while some oligotrophic bacteria including Armatimonadetes, Chloroflexi, Firmicutes and Gemmatimonadetes showed the opposite changing patterns (Table S4). These findings indicated that those specific bacterial taxa response patterns were in line with general life-history strategies, and it was proposed that copiotrophic bacteria would exhibit higher growth rates than oligotrophic taxa when the $\mathrm{N}$ resources are increased after long-term continuous cropped alfalfa in this study. In addition, we observed that Actinobacteria, Chloroflexi, Acidobacteria and Alphaproteobacteria comprised $67.94 \%$ of the total sequences and those bacterial phyla could explain $50.86 \%$ of the bacterial community variations in response to the consecutive cropping. Intriguingly, among these changed phyla, the relative abundance of Chloroflexi was less than that of Alphaproteobacteria and Acidobacteria, but the contribution in shaping the variation conducted by Chloroflexi was $20.88 \%$ and $95.87 \%$ higher than that of Alphaproteobacteria and Acidobacteria, respectively. This finding inferred that a relatively lower abundance of the specific bacterial taxa could serve a high contribution in shifting the bacterial community responds to the continuous cropping.

In addition, we observed that the relative abundances of Arthrobacter, Bacillus, Burkholderiaceae and Microbacterium showed a successively decreasing tendency with continuous cropping years. Given that several bacterial species within those genera have been demonstrated to be beneficial bacteria that have the ability to solubilize phosphorus (Sharma et al., 2013; Panhwar et al., 2014) and potassium (Sheng and He 2006; Sugumaran and Janarthanam, 2007). In addition, many species within Bacillus have been confirmed to have the ability to improve plant growth and/or to suppress the growth of soil-borne pathogens (Zhang et al., 2011; Islam et al., 2016). Thus, the decline in the abundance of these potential beneficial bacteria might be associated with soil sickness after long-term continuous cropping. Furthermore, we detected that the abundance of some nitrogen fixation bacteria including Bradyrhizobium and Mesorhizobium obviously increased with successive continuous cropping years. Similar changing patterns of the relative abundance of Bradyrhizobium were also found in another leguminous crop of soybean after 10 years of continuous cropping (Liu et al., 2019). However, the numbers of relative abundance of Bradyrhizobium and the degree of increase after long-term continuous cropping in alfalfa were much lower than those of soybean in north-east China (Liu et al., 2019), inferring that the responses of specific functional guilds of Bradyrhizobium may differ among different leguminous crops.
4.4 Long-term continuous cropping of alfalfa simplified bacterial cooccurrence network

Microorganisms coexisting in complex networks provide an indepth comprehension of the underlying the properties of the microbial network structures and their putative keystone species to reflect their ecological processes (Barberan et al., 2012; Chow et al., 2014). We observed that continuous cropping of alfalfa exerted a remarkable impact on bacterial networks regarding network size, number of links, average clustering coefficient (avgCC), geodesic distance (GD) and connectivity (avgK), but not on the modularity between less (L10) and more (M10) than 10 years of continuously cropped alfalfa (Table 2). Specifically, the decrease in the number of the nodes and links in M10, which suggests that long-term continuous cropping treatment reduced the complexity of the bacterial networks. The decrease in stability of M10 was likely attributed to bacterial diversity loss and community structure simplifications caused by long-term continuous cropping (Shi et al., 2016; Hu et al., 2020). In addition, the higher connectivity and lower geodesic distance in L10 indicated that the bacterial network of the nodes affected with each other could be more efficient or intense in short-term continuous cropping when the external environment was perturbed (Deng et al., 2012; He et al., 2017).

The nodes of those putative keystone species were defined as generalists with module hubs and connectors, respectively, which are usually considered as gatekeepers to maintain ecosystem functions and play important roles in biogeochemical cycling (Lynch and Neufeld, 2015; Jiang et al., 2016). We observed that most of the bacterial keystone species including Actinomycetales, Nocardioides, Blastococcus and Amycolatopsis were classified as Actinobacteria in M10, which broadly colonized in the agriculture soils, and had the ability to decompose an extensive range of substrates as a carbon source (Aislabie et al., 2006). Since Banerjee et al. (2016) has recently documented that the decomposition of soil organic matter is mainly and well associated with keystone taxa but not with microbial diversity, we supposed that such bacteria annotated as keystone species in long-term continuous cropping might be associated with allelochemical decomposition that was generated in continuous cropping systems (Kong et al., 2008; Guo et al., 2011). In contrast, several species closely related to Chloroflexi were defined as module hubs and connectors in $\mathrm{L} 10$ and $\mathrm{M} 10$, respectively, which belonged to slower-growing bacteria with lower carbon availability preferred to oligotrophic conditions. These kinds of oligotrophic populations serve as keystone species, inferring that these bacteria might be recruited to resist the relative deficiencies in available nutrients caused by continuous cropping (Wang et al., 2020). Intriguingly, the nodes annotated as generalists in M10 were present as specialists in L10 treatment (Table S6), which indicated that the same OTUs 
exhibited different roles in response to the continuous cropping chronosequence. These results indicated that monocropping of alfalfa remarkably altered the bacterial network structures, key populations as well as the topological roles of individual OTUs.

\section{Conclusions}

In summary, our results revealed that continuous cropping exerted substantial impacts on the bacterial diversity and structure and complex cooccurrence networks. Specifically, 10 years of continuous cropping of alfalfa may be a cutoff point in regulating the shifts in soil bacterial community structures, and these variations were mainly associated with soil $\mathrm{pH}$, TP and AP contents. Long-term continuous cropping significantly decreased the relative abundance of copiotrophic bacteria of Actinobacteria and Gammaproteobacteria, but increased the relative abundances of those oligotrophic bacteria including Armatimonadetes, Chloroflexi, Firmicutes and Gemmatimonadetes, suggesting that these changing patterns were in line with general $\mathrm{r}$ - and K-selected life-history strategies, with copiotrophic bacteria exhibiting higher growth rates than the oligotrophic taxa when the $\mathrm{N}$ resources were increased after long-term continuously cropped alfalfa. Furthermore, the decline in the abundance of beneficial bacteria such as Arthrobacter, Bacillus, Burkholderiaceae and Microbacterium was involved in the process of phosphate and potassium cycling, indicating that they might be associated with the continuous cropping obstacles. Overall, our results demonstrated that long-term continuous cropping diminished the stability and complexity of the bacterial community structures through the reduction in bacterial species diversity and its interaction.

\section{Acknowledgments}

This work was supported by grants from National Key Research and Development Program of China (2017YFD0200604), Key Research Program of Frontier Sciences, CAS (ZDBS-LYDQC017), the National Natural Science Foundation of China (41671251), Earmarked Fund for China Agriculture Research System (CR34), Heilongjiang Provincial Natural Science Foundation of China (D2018009), the Grass-field Rotation Scientist Studio of Heilongjiang Province (202004), Outstanding Youth Fund of Heilongjiang Academy of Agricultural Sciences (2020JCQN003), and Youth Innovation Promotion Association, CAS (2017276).

\section{Conflict of interest}

The authors declare no conflict of interest

\section{References}

Aislabie, J.M., Chhour, K., Saul, D.J., Miyauchi, S., Ayton, J.,
Paetzold, R.F., Balks, M.R., 2006. Dominant bacteria in soils of Marble Point and Wright Valley, Victoria Land, Antarctica. Soil Biology \& Biochemistry 38, 3041-3056.

Bagavathiannan, M.V., Gulden, R.H., Van Acker, R.C., 2011. Occurrence of alfalfa (Medicago sativa L.) populations along roadsides in southern Manitoba, Canada and their potential role in intraspecific gene flow. Transgenic Research 20, 397-407.

Bai, L., Cui, J.Q., Jie, W., Cai, B.Y., 2015. Analysis of the community composition of rhizosphere fungi in soybeans continuous cropping fields. Microbiological Research 180, 49-56.

Banerjee, S., Kirkby, C.A., Schmutter, D., Bissett, A., Kirkegaard, J. A., Richardson, A.E., 2016. Network analysis reveals functional redundancy and keystone taxa amongst bacterial and fungal communities during organic matter decomposition in an arable soil. Soil Biology \& Biochemistry 97, 188-198.

Barberan, A., Bates, S.T., Casamayor, E.O., Fierer, N., 2012. Using network analysis to explore co-occurrence patterns in soil microbial communities. ISME Journal 6, 343-351.

Biddle, J.F., Fitz-Gibbon, S., Schuster, S.C., Brenchley, J.E., House, C.H., 2008. Metagenomic signatures of the Peru Margin subseafloor biosphere show a genetically distinct environment. Proceedings of the National Academy of Sciences of the United States of America 105, 10583-10588.

Chen, S., Qi, G.F., Luo, T., Zhang, H.C., Jiang, Q.K., Wang, R., Zhao, X.Y., 2018. Continuous cropping tobacco caused variance of chemical. Land Degradation \& Development 29, 4106-4120.

Chow, C.E.T., Kim, D.Y., Sachdeva, R., Caron, D.A., Fuhrman, J.A., 2014. Top-down controls on bacterial community structure: microbial network analysis of bacteria, T4-like viruses and protists. ISME Journal 8, 816-829.

Chu, H.Y., Fierer, N., Lauber, C.L., Caporaso, J.G., Knight, R., Grogan, P., 2010. Soil bacterial diversity in the Arctic is not fundamentally different from that found in other biomes. Environmental Microbiology 12, 2998-3006.

Cleveland, C.C., Liptzin, D., 2007. C:N:P stoichiometry in soil: Is there a Redfield ratio for the microbial biomass? Biogeochemistry 85, 235-252.

Degrune, F., Dufrêne, M., Colinet, G., Massart, S., Taminiau, B., Bodson, B., Hiel, M.P., Daube, G., Nezer, C., Vandenbol, M., 2015. A novel sub-phylum method discriminates better the impact of crop management on soil microbial community. Agronomy for Sustainable Development 35, 1157-1166.

Deng, Y., Jiang, Y.H., Yang, Y., He, Z., Luo, F., Zhou, J., 2012. Molecular ecological network analyses. BMC Bioinformatics 13, 113.

Edgar, R.C., Haas, B.J., Clemente, J.C., Quince, C., Knight, R., 2011. UCHIME improves sensitivity and speed of chimera detection. Bioinformatics (Oxford, England) 27, 2194-2200.

Fierer, N., Jackson, R.B., 2006. The diversity and biogeography of soil bacterial communities. Proceedings of the National Academy of Sciences of the United States of America 103, 626-631.

Gentry, L.F., Ruffo, M.L., Below, F.E., 2013. Identifying factors controlling the continuous corn yield penalty. Agronomy Journal 105, 295-303.

Guo, Z.Y., Kong, C.H., Wang, J.G., Wang, Y.F., 2011. Rhizosphere isoflavones (daidzein and genistein) levels and their relation to the 
microbial community structure of mono-cropped soybean soil in field and controlled conditions. Soil Biology \& Biochemistry 43, 2257-2264.

He, D., Shen, W., Eberwein, J., Zhao, Q., Ren, L., Wu, Q.L., 2017. Diversity and co-occurrence network of soil fungi are more responsive than those of bacteria to shifts in precipitation seasonality in a subtropical forest. Soil Biology \& Biochemistry 115, 499-510.

Hu, X.J., Liu, J.J., Yu, Z.H., Yao, Q., Zhang, W., Mi, G., Liang, A.Z., Li, L.J., Chen, X.L., Jin, J., 2020. Liu., X.B., Wang, G.H., 2020. Continuous cropping of soybean induced a more fluctuating fungal network and intensive pathogenic fungal interactions in a Mollisol of Northeast China. Soil Science Society of America Journal 84, 1-9.

Huang, L.F., Song, L.X., Xia, X.J., Mao, W.H., Shi, K., Zhou, Y.H., Yu, J.Q., 2013. Plant-soil feedbacks and soil sickness: from mechanisms to application in agriculture. Journal of Chemical Ecology 39, 232-242.

Huang, Y., Xiao, X., Huang, H.Y., Jing, J.Q., Zhao, H.J., Wang, L., Long, X.E., 2018. Contrasting beneficial and pathogenic microbial communities across consecutive cropping fields of greenhouse strawberry. Applied Microbiology and Biotechnology 2018, 57175729.

Islam, S., Akanda, A.M., Prova, A., Islam, M.T., Hossain, M.M., 2016. Isolation and identification of plant growth promoting rhizobacteria from cucumber rhizosphere and their effect on plant growth promotion and disease suppression. Frontiers in Microbiology 6, 1360.

Jiang, J.P., Xiong, Y.C., Jia, Y., Li, F.M., Xu, J.Z., Jiang, H.M., 2007. Soil quality dynamics under successional alfalfa field in the semiarid loess plateau of northwestern China. Arid Land Research and Management 21, 287-303.

Jiang, Y., Liang, Y., Li, C., Wang, F., Sui, Y., Suvannange, N., Zhou, J., Sun, B., 2016. Crop rotations alter bacterial and fungal diversity in paddy soils across East Asia. Soil Biology \& Biochemistry 95, 250-261.

Kong, C.H., Wang, P., Zhao, H., Xu, X.H., Zhu, Y.D., 2008. Impact of allelochemical exuded from allelopathic rice on soil microbial community. Soil Biology \& Biochemistry 40, 1862-1869.

Li, C.G., Li, X.M., Kong, W.D., Wu, Y., Wang, J.G., 2010. Effect of monoculture soybean on soil microbial community in the Northeast China. Plant and Soil 330, 423-433.

Li, W.H., Liu, Q.Z., 2019. Changes in fungal community and diversity in strawberry rhizosphere soil after 12 years in the greenhouse. Journal of Integrative Agriculture 18, 677-687.

Li, Y., Huang, M., 2008. Pasture yield and soil water depletion of continuous growing alfalfa in the Loess Plateau of China. Agriculture, Ecosystems \& Environment 124, 24-32.

Liu, C.X., Zhao, X.R., Lin, Q.M., Li, G.T., 2019. Decrease in diversity and shift in composition of the soil bacterial community were closely related to high available phosphorus in agricultural Fluvisols of North China. Acta Agriculturæ Scandinavica. Section B, Soil and Plant Science 69, 618-630.

Liu, J.J., Yao, Q., Li, Y.S., Zhang, W., Mi, G., Chen, X.L., Yu, Z.H., Wang, G.H., 2019. Continuous cropping of soybean alters the bulk and rhizospheric soil fungal communities in a Mollisol of Northeast
PR China. Land Degradation \& Development 30, 1725-1738.

Liu, J.J., Yu, Z.H., Yao, Q., Hu, X.J., Zhang, W., Mi, G., Chen, X.L., Wang, G.H., 2017. Distinct soil bacterial communities in response to the cropping system in a Mollisol of northeast China. Applied Soil Ecology 119, 407-416.

Liu, X., Zhang, J.L., Gu, T.Y., Zhang, W.M., Shen, Q.R., Yin, S.X., Qi, H.Z., 2014. Microbial community diversities and taxa abundances in soils along a seven-year gradient of potato monoculture using high throughput pyrosequencing approach. PLoS One 9, e86610.

Liu, X.B., Li, Y.S., Han, B.J., Zhang, Q.Y., Zhou, K.Q., Zhang, X.Y., Hashemi, M., 2012. Yield response of continuous soybean to oneseason crop disturbance in a previous continuous soybean field in Northeast China. Field Crops Research 138, 52-56.

Luo, C.G., Deng, Y.W., Inubushi, K., Liang, J., Zhu, S.P., Wei, Z.Y., Guo, X.B., Luo, X.P., 2018. Sludge biochar amendment and alfalfa revegetation improve soil physicochemical properties and increase diversity of soil microbes in soils from a rare earth element mining wasteland. International Journal of Environmental Research and Public Health 15, 965.

Lynch, M.D., Neufeld, J.D., 2015. Ecology and exploration of the rare biosphere. Nature Reviews. Microbiology 13, 217-229.

Magoč, T., Salzberg, S.L., 2011. FLASH: fast length adjustment of short reads to improve genome assemblies. Bioinformatics (Oxford, England) 27, 2957-2963.

Panhwar, Q.A., Naher, U.A., Jusop, S., Othman, R., Latif, M.A., Ismail, M.R., 2014. Biochemical and molecular characterization of potential phosphate solubilizing bacteria in acid sulfate soils and their beneficial effects on rice growth. PLoS One 9, e97241.

Price, M.N., Dehal, P.S., Arkin, A.P., 2009. FastTree: computing large minimum evolution trees with profiles instead of a distance matrix. Molecular Biology and Evolution 26, 1641-1650.

Prosser, J.I., 2019. Exploring soil microbial communities: Opportunities for soil ecology research. Soil Ecology Letters 1, 1-2.

R Development Core Team, 2010. R: a Language and Environment for Statistical Computing. R Foundation for Statistical Computing, Vienna, Austria URL.

Ren, X.L., Jia, Z.K., Wan, S.M., Han, Q.F., Chen, X.L., 2011. The long-term effects of alfalfa on soil water content in the Loess Plateau of northwest China. African Journal of Biotechnology 10 , $4420-4427$.

Rosenzweig, N., Tiedje, J.M., Quensen, J.F. III, Meng, Q.X., Hao, J. J.J., 2012. Microbial communities associated with potato common scab-suppressive soil determined by pyrosequencing analyses. Plant Disease 96, 718-725.

Sharma, S.B., Sayyed, R.Z., Trivedi, M.H., Gobi, T.A., 2013. Phosphate solubilizing microbes: Sustainable approach for managing phosphorus deficiency in agricultural soils. SpringerPlus 2, 587.

Sheng, X.F., He, L.Y., 2006. Solubilization of potassium-bearing minerals by a wild-type strain of Bacillus edaphicus and its mutants and increased potassium uptake by wheat. Canadian Journal of Microbiology 52, 66-72.

Shi, S., Nuccio, E.E., Shi, Z.J., He, Z., Zhou, J., Firestone, M.K., 2016. The interconnected rhizosphere: High network complexity dominates rhizosphere assemblages. Ecology Letters 19, 926- 
936.

Strickland, M.S., Lauber, C., Fierer, N., Bradford, M.A., 2009. Testing the functional significance of microbial community composition. Ecology 90, 441-451.

Sugumaran, P., Janarthanam, B., 2007. Solubilization of potassium containing minerals by bacteria and their effect on plant growth. World Journal of Agricultural Sciences 3, 350-355.

Tan, Y., Cui, Y.S., Li, H.Y., Kuang, A.X., Li, X.R., Wei, Y.L., Ji, X.L., 2017. Diversity and composition of rhizospheric soil and root endogenous bacteria in Panax notoginseng during continuous cropping practices. Journal of Basic Microbiology 57, 337-344.

Tsiafouli, M.A., Thebault, E., Sgardelis, S.P., de Ruiter, P.C., van der Putten, W.H., Birkhofer, K., Hemerik, L., de Vries, F.T., Bardgett, R.D., Brady, M.V., Bjornlund, L., Jørgensen, H.B., Christensen, S., Hertefeldt, T.D., Hotes, S., Gera Hol, W.H., Frouz, J., Liiri, M., Mortimer, S.R., Setälä, H., Tzanopoulos, J., Uteseny, K., Pižl, V., Stary, J., Wolters, V., Hedlund, K., 2015. Intensive agriculture reduces soil biodiversity across Europe. Global Change Biology 21, 973-985.

Udom, B.E., Ogunwole, J.O., 2015. Soil organic carbon, nitrogen, and phosphorus distribution in stable aggregates of an Ultisol under contrasting land use and management history. Journal of Plant Nutrition and Soil Science 178, 460-467.

Venter, Z.S., Jacobs, K., Hawkins, H.J., 2016. The impact of crop rotation on soil microbial diversity: a meta-analysis. Pedobiologia 59, 215-223.

Wang, H., Li, X., Li, X., Wang, J., Li, X., Guo, Q., Yu, Z., Yang, T., Zhang, H., 2020. Long-term no-tillage and different residue amounts alter soil microbial community composition and increase the risk of maize root rot in northeast China. Soil \& Tillage Research 196, 104452

Wei, W., Xu, Y.L., Li, S.X., Zhu, L., Song, J., 2015. Developing suppressive soil for root diseases of soybean with continuous longterm cropping of soybean in black soil of northeast China. Acta Agriculturæ Scandinavica. Section B, Soil and Plant Science 65, 279-285.

Xiong, W., Zhao, Q.Y., Zhao, J., Xun, W.B., Li, R., Zhang, R.F., Wu, H.S., Shen, Q.R., 2015. Different continuous cropping spans significantly affect microbial community membership and structure in a vanilla-grown soil as revealed by deep pyrosequencing Microbial Ecology 70, 209-218.

Xuan, D.T., Guong, V.T., Rosling, A., Alström, S., Chai, B., Högberg, N., 2012. Different crop rotation systems as drivers of change in soil bacterial community structure and yield of rice, Oryza sativa. Biology and Fertility of Soils 48, 217-225.

Yao, Q., Xu, Y.X., Liu, X.F., Liu, J.J., Huang, X.Y., Yang, W.G., Yang, Z., Lan, L., Zhou, J.M., Wang, G.H., 2019. Dynamics of soil properties and fungal community structure in continuous cropped alfalfa fields in Northeast China. PeerJ 7, e7127.

Zarjani, J.K., Aliasgharzad, N., Oustan, S., Emadi, M., Ahmadi, A., 2013. Isolation and characterization of potassium solubilizing bacteria in some Iranian soils. Archives of Agronomy and Soil Science 59, 1713-1723.

Zhang, J., Wang, Q., Xiao, Y., Pang, X. P., Jia, T.T., Song, R., Liu, $H . X ., 2016$. Effects of alternate furrow irrigation on the biomass allocation and water use efficiency of alfalfa. Acta Prataculturae Sinica 25, 164-171 (in Chinese).

Zhang, N., Wu, K., He, X., Li, S.Q., Zhang, Z.H., Shen, B.A., Yang, X.M., Zhang, R.F., Huang, Q.W., Shen, Q.R., 2011. A new bioorganic fertilizer can effectively control banana wilt by strong colonization with Bacillus subtilis N11. Plant and Soil 344, 8797.

Zhang, W., Long, X., Huo, X., Chen, Y., Lou, K., 2013. 16S rRNAbased PCR-DGGE analysis of actinomycete communities in fields with continuous cotton cropping in Xinjiang, China. Microbial Ecology 66, 385-396.

Zhao, Q.Y., Xiong, W., Xing, Y.Z., Sun, Y., Lin, X.J., Dong, Y.P., 2018. Long-term cofee monoculture alters soil chemical properties and microbial communities. Scientific Reports 8, 6116.

Zhong, Y., Wang, J., Song, Y., Liang, Y., Li, G., 2012. Microbial community and functional genes in the rhizosphere of alfalfa in crude oil-contaminated soil. Frontiers of Environmental Science \& Engineering 6, 797-805.

Zhou, X.G., Gao, D.M., Liu, J., Qiao, P.G., Zhou, X.L., Lu, H.B., Wu, X., Liu, D., Jin, X., Wu, F.Z., 2014. Changes in rhizosphere soil microbial communities in a continuously monocropped cucumber (Cucumis sativus L.) system. European Journal of Soil Biology 60, 1-8.

Zhou, X.G., Wang, Z.L., Jia, H.T., Li, L., Wu, F.Z., 2018. Continuously monocropped jerusalem artichoke changed soil bacterial community composition and ammonia-oxidizing and denitrifying bacteria abundances. Frontiers in Microbiology 9, 705.

Zhu, Y.B., Shi, F.Y., Zhang, R.J., Wu, Y.P., 2014. Comparison of bacterial diversity in rotational and continuous soybean cropping soil in Heilongjiang. Acta Phytophylacica Sinica 41, 403-409 (in Chinese). 